\title{
MAGIC-II's central pixel system for simultaneous optical and gamma-ray observation
}

\author{
John Hoang, ${ }^{\text {a,* Tarek Hassan, }}{ }^{\text {b }}$ Luis Angel Tejedor, ${ }^{a}$ Juan Abel Barrio, \\ Marcos López, ${ }^{a}$ David Fink, ${ }^{c}$ Martin Will, ${ }^{c}$ and Jose Luis Contreras ${ }^{a}$ \\ ${ }^{a}$ Universidad Complutense de Madrid, Institute of Particle and Cosmos Physics, \\ Departamento de Estructura de la Materia, Física Térmica y Electrónica, Madrid, Spain \\ ${ }^{\mathrm{b}}$ Deutsches Elektronen-Synchrotron, Zeuthen, Germany \\ ${ }^{\mathrm{c}}$ Max Planck Institute for Physics, Munich, Germany
}

\begin{abstract}
The MAGIC telescopes are a system of two imaging atmospheric Cherenkov telescopes designed to observe very-high-energy $\gamma$ rays. MAGIC utilizes a large reflective surface and photodetectors with ultrafast time response to capture Cherenkov photons. These features, together with the dedicated system installed in the central photomultiplier tube of their camera, so-called central pixel system (CPS), turn MAGIC into a suitable telescope to study high-speed optical astronomy in the millisecond (ms) regime. We report on the status of the CPS currently mounted in the MAGIC-II camera, its performance and calibration to demonstrate the sensitivity of MAGIC-II to ms optical pulses, for both transient and periodic signals, and discuss its potential over several science cases. (c) The Authors. Published by SPIE under a Creative Commons Attribution 4.0 Unported License. Distribution or reproduction of this work in whole or in part requires full attribution of the original publication, including its DOI. [DOI: 10.1117/1.JATIS.6.3.036002]
\end{abstract}

Keywords: imaging atmospheric Cherenkov telescopes; gamma-ray astronomy; optical astronomy; photomultipliers; optical pulsations; time-domain astronomy.

Paper 20016 received Feb. 6, 2020; accepted for publication Aug. 13, 2020; published online Aug. 28, 2020.

\section{Introduction}

Imaging atmospheric Cherenkov telescopes (IACTs) are instruments designed to indirectly detect very-high-energy (VHE) cosmic and $\gamma$ rays. The MAGIC telescopes are a system of two IACTs located at La Palma, Spain. Each telescope consists of a 17-m-diameter parabolic tessellated mirror that focuses the light onto a camera placed at the focal plane of the telescope. Each camera consists of a matrix of 1039 fast-response photomultiplier tubes (PMTs) designed to measure the short (ns scale) Cherenkov light pulses produced within extended atmospheric showers. The first telescope (MAGIC-I) was built in 2004 and operated for five years in stand-alone mode. The second MAGIC telescope (MAGIC-II), at a distance of $85 \mathrm{~m}$ from the first, started taking data from July 2009. In 2012, the two telescopes underwent a major upgrade to homogenize their cameras and electronic readout systems, and together they form the current MAGIC stereoscopic system. ${ }^{1}$

Despite the relatively modest quality of their mirrors (both in reflectivity and resolution) as compared to the usual standards in state-of-the-art optical astronomy, these telescopes have properties that make them competitive ultrafast optical telescopes: the very large size of their reflectors makes them effective "photon collectors," while strongly reducing the scintillation noise induced by atmospheric turbulence. ${ }^{2,3}$ Additionally, their PMTs are capable of monitoring photon fluxes down to extremely short timescales, several orders of magnitude faster than the readout time of charge-coupled devices (CCDs), the most common type of photodetectors used by observatories operating in the optical domain. For these reasons, IACTs have been employed in several studies other than their primary goals of searching for gamma-ray emission from astrophysical sources. For example, the idea of using Cherenkov telescopes as fast optical telescopes to measure the optical pulsations of pulsars is more than two decades old with a rich history. In 2000, the Whipple 10-m Telescope detected the optical signal from the Crab Pulsar in phase

*Address all correspondence to John Hoang, E-mail: kimhoang@ucm.es 
with the radio pulse. ${ }^{4}$ In 2004, the HEGRA CT1 reported the detection of optical pulse from the Crab Pulsar using a modified PMT after 200 s. ${ }^{5}$ Similarly, pulsed emission from the Crab Pulsar has been measured successfully by all current-generation IACT instruments, including MAGIC, ${ }^{6}$ HESS, ${ }^{7}$ and VERITAS. ${ }^{8}$ Other employments of Cherenkov telescopes include meteor studies, ${ }^{9}$ optical search for extraterrestrial intelligence, ${ }^{10,11}$ their use as stellar intensity interferometers, ${ }^{12}$ or directly measuring stellar angular diameters. ${ }^{13}$

In 2006, the MAGIC collaboration implemented a central pixel system (CPS) in MAGIC-I ${ }^{6}$ to take advantage of MAGIC capabilities as a fast optical detector. After the camera upgrades in 2012, the CPS in MAGIC-I was decommissioned and a new CPS was implemented in the MAGIC-II camera. This new system allows one to perform simultaneous optical and VHE observations, turning MAGIC into a suitable instrument to search for multiwavelength counterparts of fast transients in both the VHE and optical regimes.

This work is structured as follows. In Sec. 2, the technical aspects of the CPS of MAGIC-II are described, including the new photosensor with improved detection efficiency, the high bandwidth electro-to-optical transmission system, and the modified digitizing system. In Sec. 3, we demonstrate the sensitivity of the CPS to millisecond (ms) pulses both for periodic and isolated signals. Section 4 discusses several science cases where the fast timing capabilities of MAGIC can be further exploited to make major discoveries.

\section{Central Pixel System}

The CPS consists of a fully modified photosensor-to-readout chain exploiting the PMT located at the center of the MAGIC-II telescope camera. A standard MAGIC pixel consists of a PMT followed by a preamplifier that divides the signal in two branches: a so-called $\mathrm{AC}$ branch to process Cherenkov light pulses at a high bandwidth range (between 1 and $300 \mathrm{MHz}$ ) and a so-called DC branch to monitor the PMT anode current and indirectly measure the sky brightness in the observation area to prevent potential damage to the PMT while observing under high night-sky background levels. The CPS involves modifying the DC branch to increase its bandwidth from its nominal value of $8 \mathrm{~Hz}$ to over $3 \mathrm{kHz}$ in order to detect pulses with ms duration. The output of this new DC branch is fed to both the standard DC monitoring system and to an additional optical transmitter that sends the signal to the counting house, a building where the readout and the trigger electronics are outsourced from the telescope camera so that the camera has less weight and provides generous dimensions for easy maintenance. There, it is converted and adapted to be readout by the standard MAGIC data acquisition (DAQ) system (called from here on Domino readout) and also delivered to a dedicated CPS computer running Linux for special data readout and processing. In this way, the standard functionality of the pixel is maintained (Cherenkov photon observation and PMT anode current monitoring) while the additional possibility to observe ms optical transients is incorporated. The block diagram for the CPS is shown in Fig. 1.

\subsection{Photosensor}

The photosensor of the CPS is identical to the rest of the pixels in the MAGIC-II camera. They are PMTs from Hamamatsu (super-bialkali R10408), each with 25.4-mm diameter,

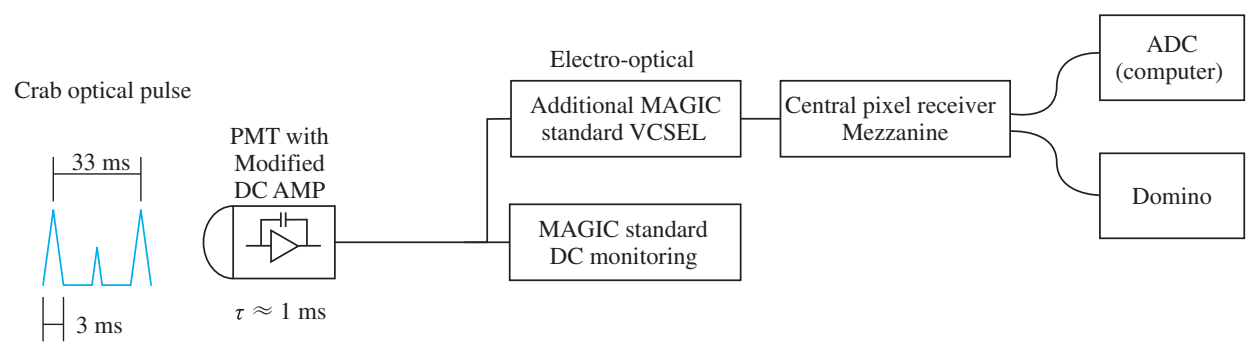

Fig. 1 CPS block diagram, showing the upgraded DC branch. The AC branch, used to process pulses of Cherenkov photons associated with $\gamma$-ray induced showers, is not drawn in the diagram. 


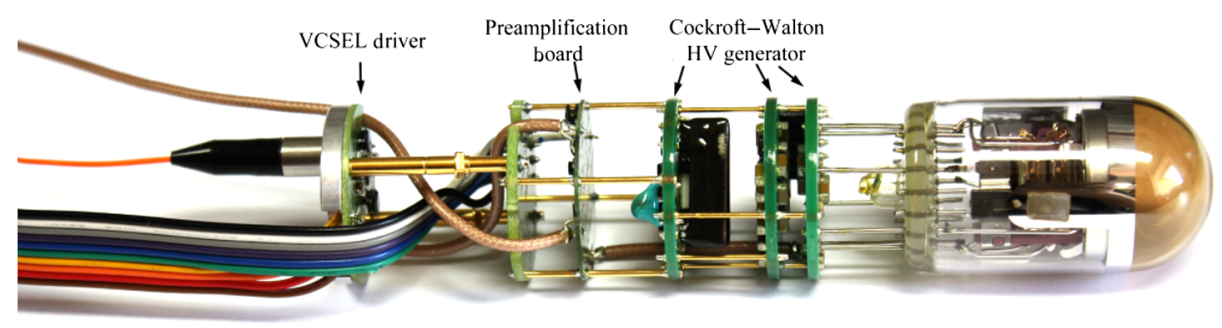

Fig. 2 The PMT of the CPS assembled with its high voltage generator, a preamplifier, and a VCSEL driver. These electronic boards control and process the sensor's response into optical output signal ready to transmit via multimode optical fiber.

a hemispherical photocathode, and 6 dynodes. ${ }^{1}$ Relative to the PMTs of the original MAGIC-I camera (Electron Tubes ET9116B) used for the old CPS, the new PMTs have an enhanced quantum efficiency in the blue wavelength $\left(\sim 32 \%\right.$ at $\left.330 \mathrm{~nm}^{6}\right)$. The PMTs are grouped in clusters of seven to form a modular unit to facilitate installation and maintenance. Each single cluster weighs $\sim 1 \mathrm{~kg}$, has a length of $50 \mathrm{~cm}$, and a width of $9 \mathrm{~cm}$, with a distance of $3 \mathrm{~cm}$ between the pixel centers.

Every PMT is connected to several electronic boards as seen in Fig. 2. The first three closest to the PMT come from the manufacturer of the PMT and are responsible for generating high voltage and feeding it to the PMT dynodes. The high voltage is generated by a Cockroft-Walton voltage multiplier, which is controlled by the slow-control board of the cluster.

\subsection{PMT Preamplifier}

The PMT preamplifier and DC sensing circuitry are placed in the fourth board, as shown in Fig. 2. To achieve a bandwidth spanning from $1 \mathrm{~Hz}$ to several $\mathrm{kHz}$ in the DC branch so that the fast signal (ms regime) can be detected, several passive components in this preamplification board were exchanged (Table 1). These changes are drawn in green in the schematic in Fig. 3. Figure 4 shows the bandwidth of the DC branch after the above-mentioned modifications, demonstrating the capability of observing fast $\mathrm{kHz}$ signals (reaching the $3-\mathrm{dB}$ point at $\sim 3 \mathrm{kHz}$ ). The last board attached to the mechanical structure of the PMT is the temperature sensor and driver board for the vertical-cavity surface-emitting laser (VCSEL), which is used to deliver the AC branch signal of each PMT to the counting house via the optical fiber. ${ }^{14}$

\subsection{Optical Transmitter}

The analog output signal of the AC branch from every pixel of the MAGIC camera is transmitted to the counting house using a set of optical fibers, one per pixel. As a result, the data transmission

Table 1 Values of the replaced components (shown in green in Fig. 3) for the PMT preamplifier of the CPS in order to increase the bandwidth.

\begin{tabular}{lcc}
\hline \hline Component & Original value & Replaced value \\
\hline $\mathrm{C} 1$ & $100 \mathrm{nF}$ & $10 \mathrm{nF}$ \\
$\mathrm{R} 7$ & $91 \mathrm{k} \Omega$ & $0 \Omega$ \\
$\mathrm{R} 1$ & $10 \mathrm{k} \Omega$ & $470 \mathrm{k} \Omega$ \\
$\mathrm{R} 9$ & $1 \mathrm{M} \Omega$ & $4.7 \mathrm{k} \Omega$ \\
$\mathrm{R} 10$ & $10 \mathrm{M} \Omega$ & $100 \mathrm{k} \Omega$ \\
$\mathrm{C} 9$ & $1 \mathrm{nF}$ & $100 \mathrm{pF}$ \\
\hline \hline
\end{tabular}




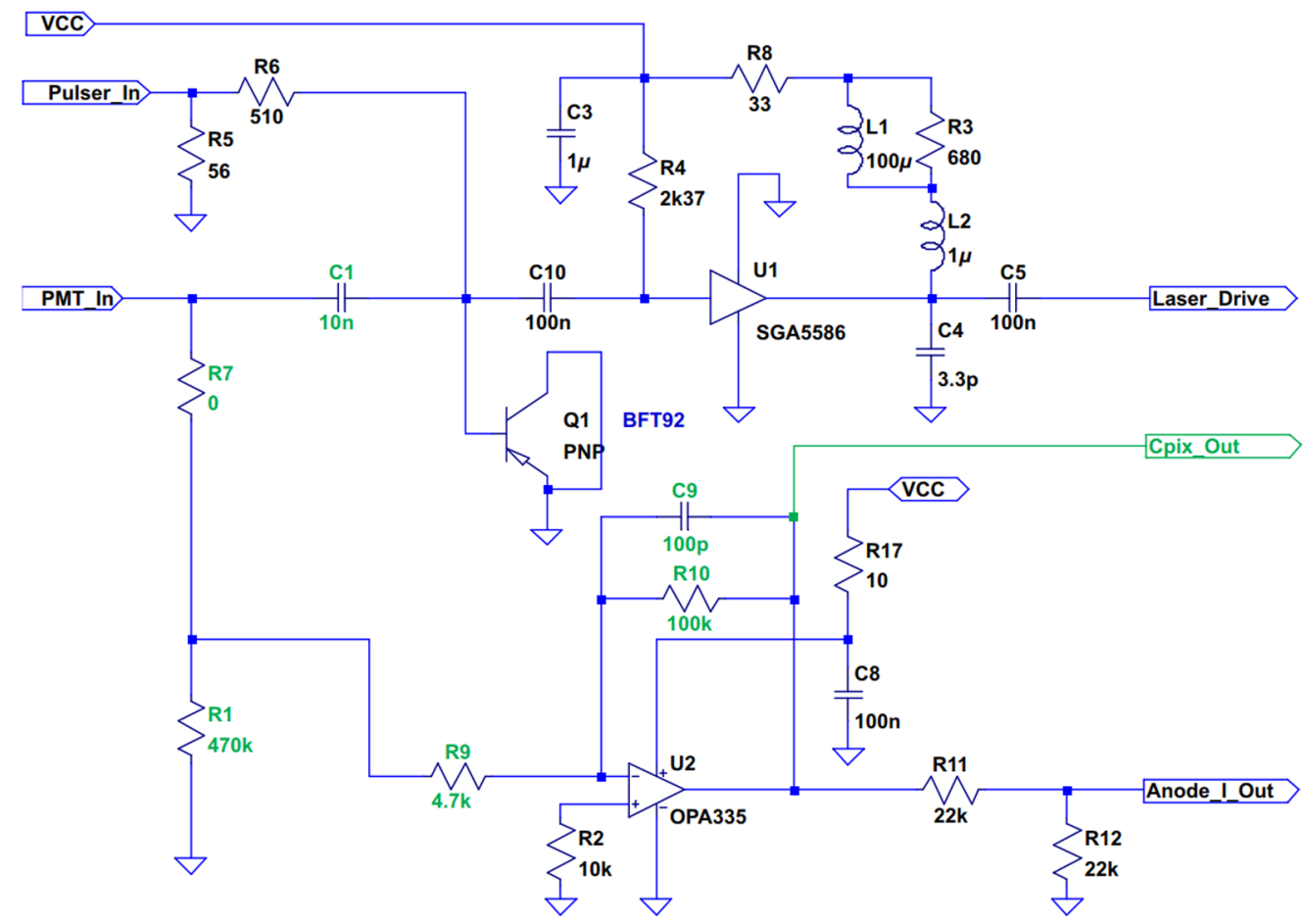

Fig. 3 Schematic of the modified PMT preamplifier. Several original components were replaced so as to increase the bandwidth up to $\mathrm{kHz}$ sampling rate. They are shown in green, and their values are given in Table 1.

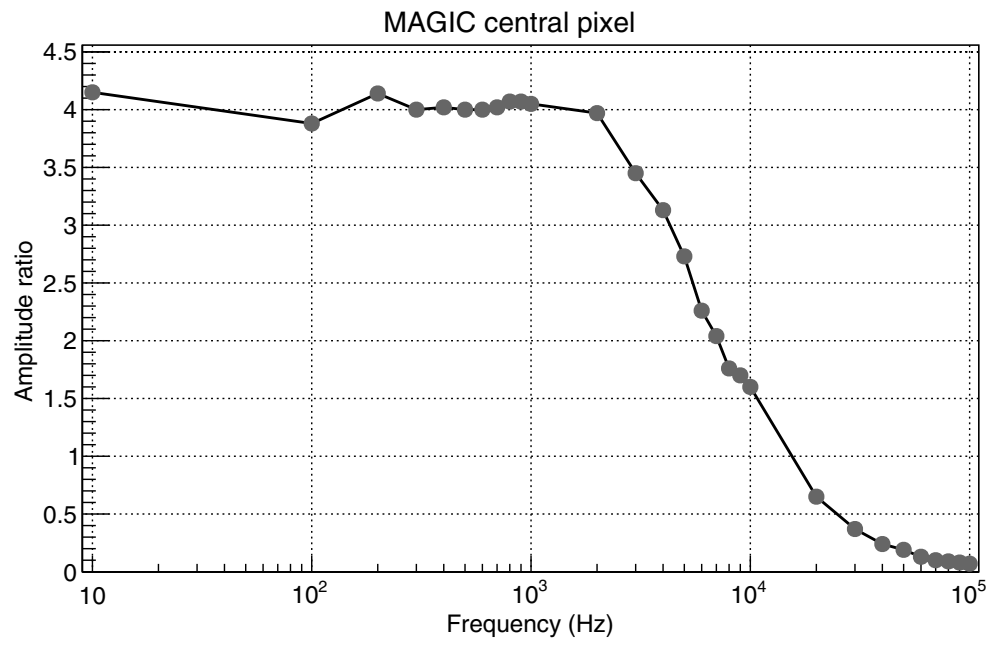

Fig. 4 Measured CPS bandwidth after modifying the DC branch, showing the achieved capability of observing fast $\mathrm{kHz}$ signals. The CPS can measure signal to $3 \mathrm{kHz}$, as seen by the $3-\mathrm{dB}$ point on the plot.

lines are both lightweight and thin. On the other hand, they are also fragile, so spare fibers were installed between camera and counting house as a redundancy measure. The CPS signal takes advantage of one of these spare fibers to deliver its analog signal to the counting house. The electrical signal is converted to an optical signal and successively transmitted by a standard VCSEL via the optical fiber. The conversion board contains a single stage consisting of an operational amplifier in a noninverting configuration driving a VCSEL laser. The schematic is shown in Fig. 5. As a precautionary measure, a Zener diode is in place to limit the output voltage of the operational amplifier to protect the VCSEL from burning in case a bright light shines on 


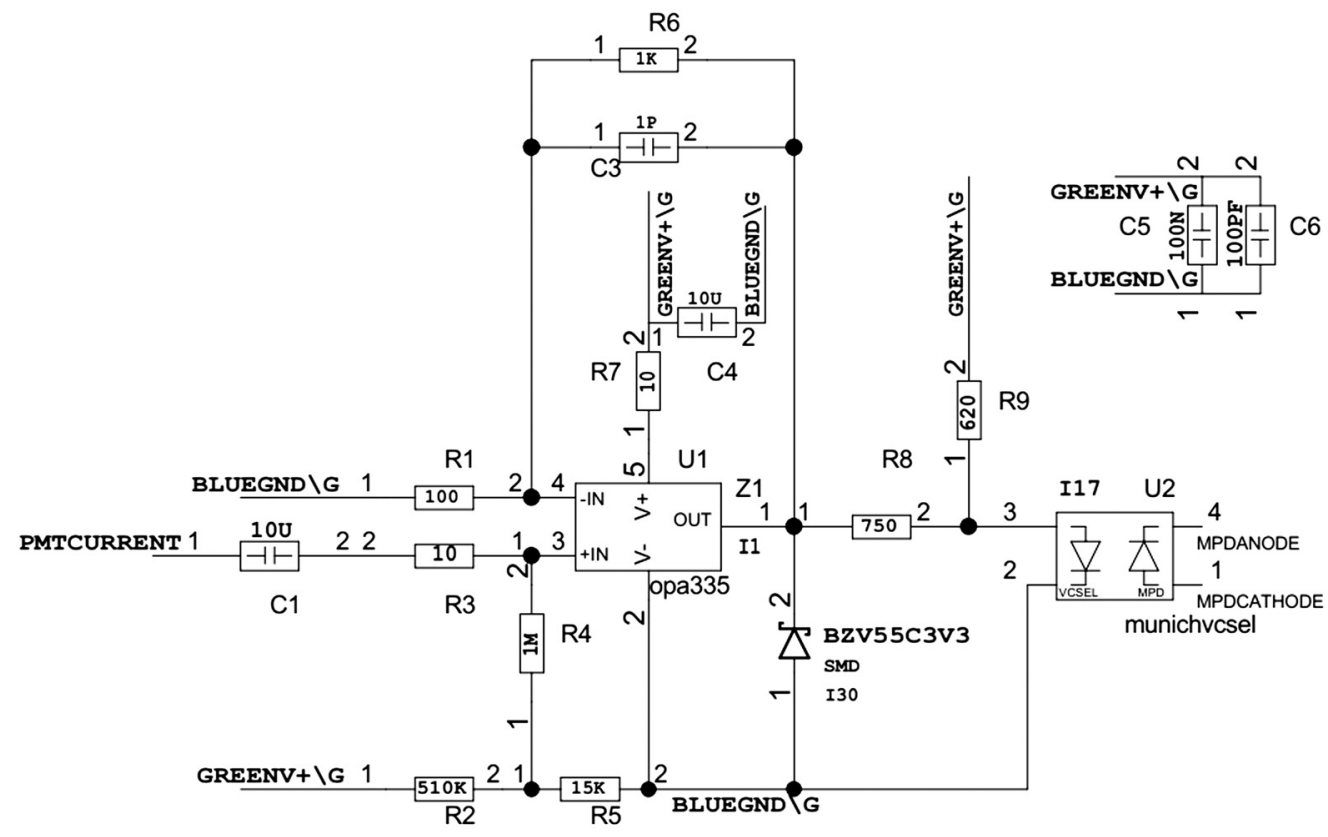

Fig. 5 Schematic of the optical transmitter.

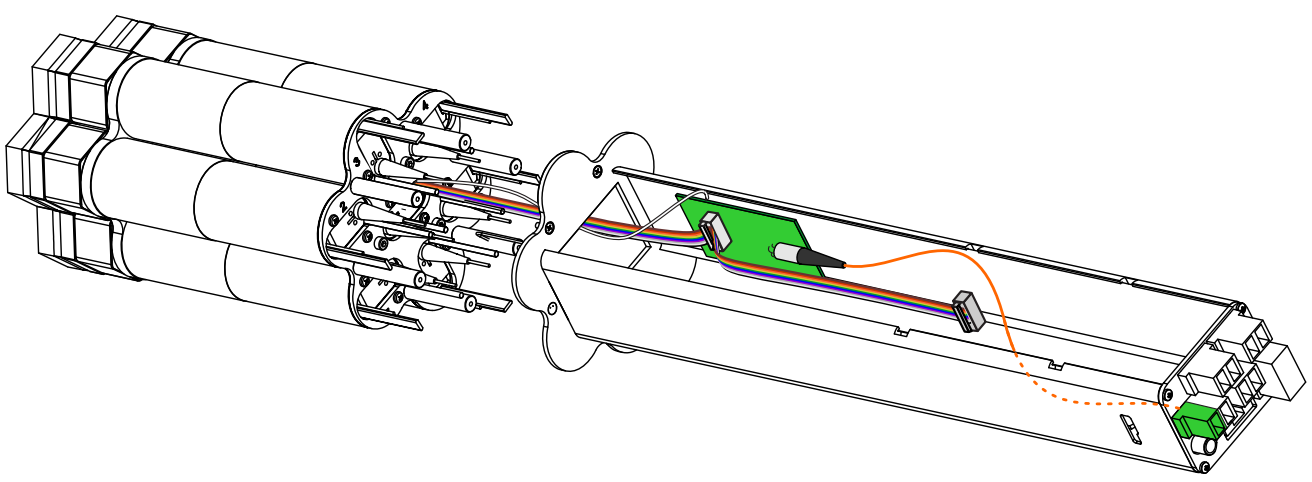

Fig. 6 MAGIC cluster hosting the CPS, the location of the optical transmitter board is shown in green. The VCSEL laser for the CPS and its corresponding optical fiber (in orange) are also highlighted.

the PMT. The power for this board is supplied by a ribbon cable attached to the central PMT. The ground and $+5 \mathrm{~V}$ DC supply-voltage signal are connected using a standard insulationdisplacement connector. It should be noted that the transmitter circuit is AC-coupled to the central pixel signal, and therefore the absolute value of the optical illumination of the PMT cannot be obtained. In Fig. 6, the board implementing the optical transmitter is shown (in green) inside the MAGIC cluster hosting the CPS. Also shown in green is the special socket to connect the optical fiber that transports the CPS optical signal.

\subsection{Optical Receiver Mezzanine}

A receiver circuit (so-called receiver board) digitizes the optical signal that arrives from the camera at the MAGIC counting house. The circuit diagram of this board is shown in Fig. 7. The low-frequency signal characteristics of the CPS output (few $\mathrm{kHz}$ ) are incompatible with the standard MAGIC receiver analog channels since they involve typical high-pass filters of several MHz. Therefore, one channel in a receiver board is replaced by the CPS optical receiver, implemented on a mezzanine board connected to a dedicated receiver board. The mezzanine 


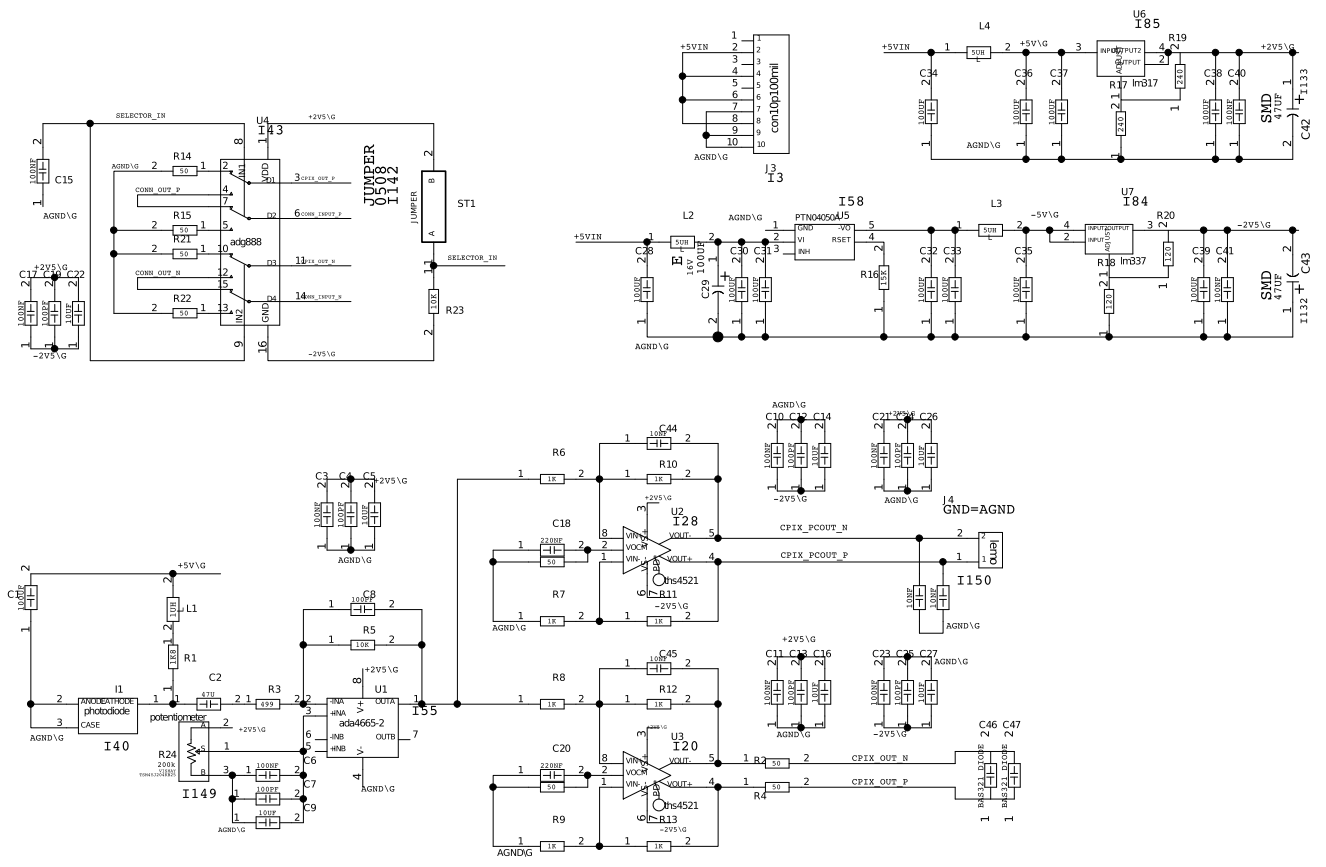

Fig. 7 Schematic of the receiver.

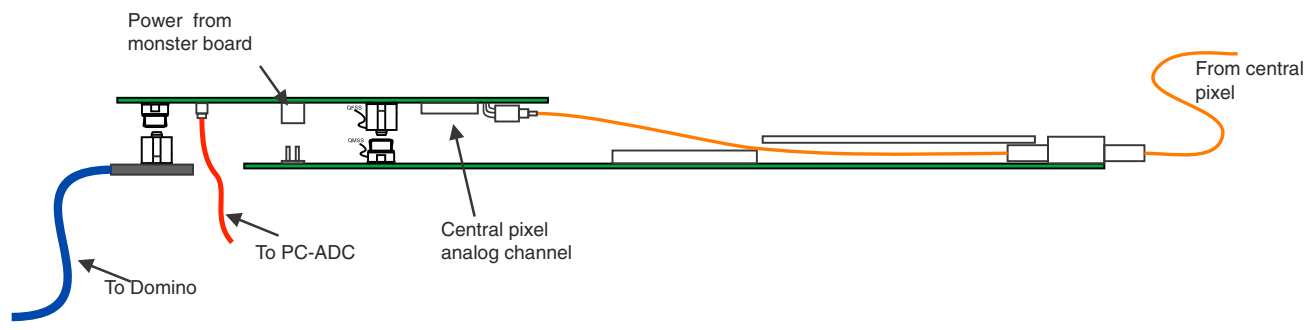

Fig. 8 Diagram of the CPS optical receiver mezzanine, connected to the programmable receiver board called monster board. The LEMO bipolar cable transporting the differential CPS optical signal to the dedicated PC-ADC readout is shown in red. The standard Cherenkov pulse is routed to the Domino readout via a separate branch and is shown in blue.

board comprises several stages: the first stage is the photodiode that converts the optical signal coming from the camera to an electric signal. With the proper DC biasing, the photodiode will produce current proportional to the incoming light. Subsequently, the signal goes through a coupling capacitor, which removes the DC component. After the signal is amplified, it is converted to differential and sent both to the Domino readout (as if it were a signal from a standard pixel) and to the CPS PC via a LEMO bipolar connector. Figure 8 shows a diagram with all the abovementioned stages and how the mezzanine is connected to the receiver board.

\subsection{CPS Digitizing System}

To achieve maximum sensitivity for the CPS, its signal is digitized in a dedicated way, independent of the standard MAGIC readout system. The differential signal produced at the optical receiver mezzanine is digitized by a National Instrument PCIe $6251 \mathrm{M}$ Series ADC card with 16-bit accuracy, named herein PC-ADC. This PC-ADC card is hosted by a dedicated computer in the MAGIC counting house, which is responsible for data taking and storing the digitized CPS signals produced by the PC-ADC.

The PC-ADC operation relies on the MAGIC timing system, specifically on its Microsemi XLi unit, which combines in a single module, a GPS receiver, and a high-precision rubidium 
oscillator, disciplined by the GPS timing signals. The Microsemi XLi module provides timing signals with accuracy and precision better than $100 \mathrm{~ns}$. In particular, a 1 pulse-per-second (PPS) signal and a 10-kHz signal (in phase with 1 PPS) are delivered to the PC-ADC. The latter is used by the PC-ADC as external reference to sample the CPS signal. To assign an absolute UTC date to each of those samples, the 1 PPS signal is also digitized with the same above-mentioned $10-\mathrm{kHz}$ external sampling reference. A program running on the computer identifies the rising edge of each 1 PPS signal and assigns to the corresponding sample a UTC date based on the NTP-synchronized time of the PC.

\section{Sensitivity to Optical Pulses}

Several tests were performed to study the optical sensitivity of MAGIC in the ms regime. Similar to other IACTs, MAGIC generally performs $\gamma$-ray observations in the so-called Wobble mode in which the source is located at an offset (0.4 deg specifically for MAGIC) away from the camera center so that the background can be estimated simultaneously over several "reflected regions" (with identical camera offset as the source location). However, because the CPS utilizes only the PMT located at the center of the camera, optical observations are required to be performed in ON mode, i.e., with the source located at the center of the camera. MAGIC can search for both $\gamma$-ray and optical signals from the source simultaneously in ON mode, but a nonstandard method to estimate the $\gamma$-ray background is required. The sampling rate of the CPS during the observations reported in this work is $10 \mathrm{kHz}$ ( 1 sample every $0.1 \mathrm{~ms})$.

\subsection{Periodic Signal: Crab Pulsar Observation}

The Crab Pulsar (PSR B0531+21) is the brightest pulsar among the few pulsars to be identified optically. Since its discovery, ${ }^{15,16}$ it has been a target of many observational campaigns over the whole electromagnetic spectrum from radio to VHE $\gamma$-ray. ${ }^{17}$ The optical Crab pulsation can be detected by the standard MAGIC DAQ. As seen in Fig. 1, the modified DC-branch output signal is also digitized in the standard MAGIC DRS4 channel. A trigger occurs due to a possible $\gamma$-ray event candidate (at the rate of $\sim 200 \mathrm{~Hz}$ ). Subsequently, using the dedicated Psearch software, the time stamp of this candidate event is assigned into the appropriate phase of the Crab Pulsar. This method provides the time stamp for both the optical CPS samples and for the VHE $\gamma$-ray events detected by MAGIC, which could play a crucial role in tagging possible coincident opticalgamma emission.

The new CPS is able to continuously sample the optical signal from the Crab Pulsar at the rate of $10 \mathrm{kHz}$. By folding the light curve of its periodic pulses, we are able to calibrate our instrument, associating the CPS measured voltage with the well-known optical flux. For this reason, the Crab Pulsar was periodically observed in $\mathrm{ON}$ mode for about $20 \mathrm{~min}$. A method equivalent to the analysis in Ref. 6 was used to obtain the phase of each sample, relying on the Crab Pulsar ephemerides provided by the Jodrell Bank Observatory. ${ }^{18,19}$ Subsequently, all phases were folded into a single phaseogram. The Crab pulsation is well reproduced in the phaseogram presented in Fig. 9(a), clearly showing its main pulse, P1, at phase 0.3 , and secondary pulse, $\mathrm{P} 2$, at phase 0.7 . Using the average flux of the Crab Pulsar in the $\mathrm{U}$ band and assuming a pulse shape equivalent to the Crab light curve taken by Aqueye, ${ }^{20}$ we can derive the relation between the voltage measured by the CPS and the optical magnitudes in the U band. Since the CPS data are affected by highfrequency noise (caused by the frequency of the DC power supply to electronics plus night-sky background), a 1-ms averaging filter is applied to estimate the RMS of the background using offsource data. Figure 9(b) shows the measured light curve of the Crab Pulsar, compared with the equivalent magnitude of 1- and 5- $\sigma$ level fluctuations coming from the background. Using this method, we can infer the sensitivity of the CPS to single optical pulses for different integration times, shown in Table 2. Even if the Crab pulses are well below the 1- $\sigma$ level of our background noise (and therefore individual Crab pulses cannot be detected), the phase-folding analysis technique significantly improves the sensitivity of the CPS.

The folded light curve is then compared to a uniform distribution using several well-known statistical tests, such as the $Z_{10}^{2}, H$, and $\chi^{2} / n d f$ tests, as described in Ref. 21. After $10 \mathrm{~s}$ of 

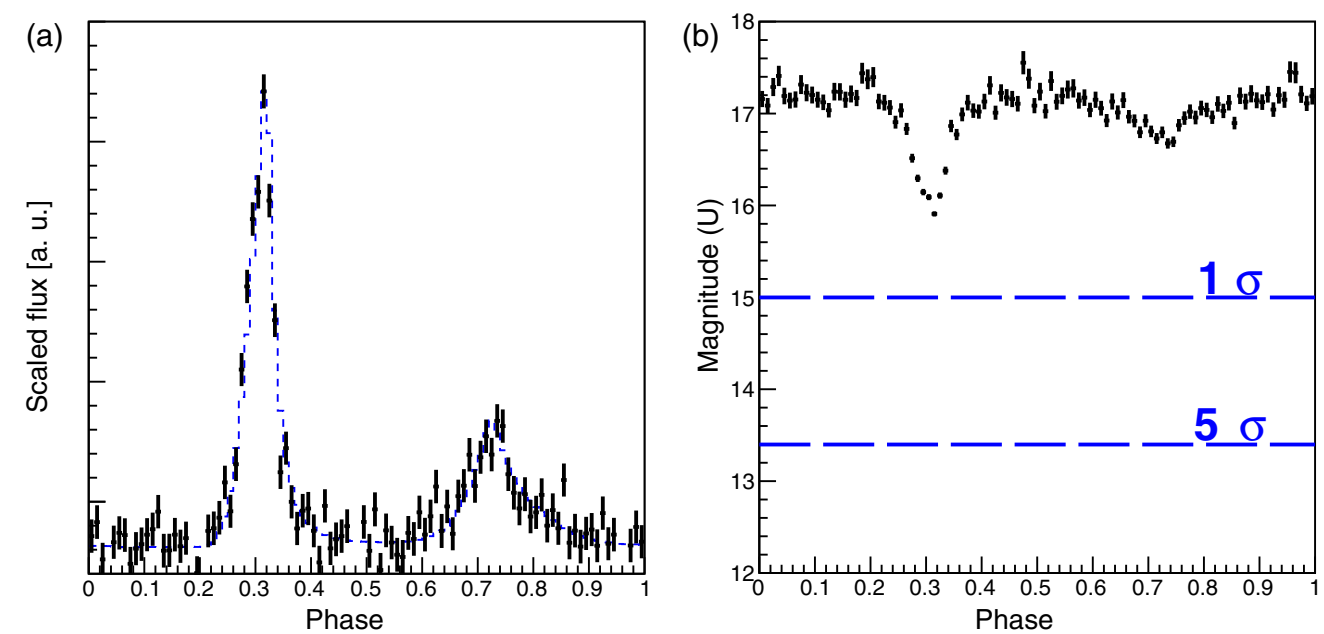

Fig. 9 (a) Folded light curve obtained from the CPS (black data with error bars) after only 5 min of observation as compared to an equally binned light curve taken by Aqueye (dashed line ${ }^{20}$ ). (b) $U$ band magnitude of the same Crab Pulsar light curve as compared with the equivalent flux levels of 1- and 5- $\sigma$ deviations measured from the background noise of the CPS.

Table 2 MAGIC sensitivity to ms pulses as a function of the integration time with a confidence level of 5 standard deviations. These numbers can be considered conservative, as they were calculated from observations close to the galactic plane, a region with high night-sky background (as described in Sec. 3.1).

\begin{tabular}{lc}
\hline \hline Integration time $(\mathrm{ms})$ & Sensitivity (mag) \\
\hline 0.1 & 12.4 \\
1 & 13.3 \\
10 & 14.4 \\
\hline \hline
\end{tabular}

observation, the significance values for $Z_{10}^{2}, H$, and $\chi^{2} / n d f$ are $6.2,6.4$, and $5.1 \sigma$, respectively. Taking the most conservative value of $5 \sigma$ among these statistical tests leads to a sensitivity of $1.6 \sigma \sqrt{t / s}$, an improvement in sensitivity relative to the old CPS (5 $\sigma$ in $20 \mathrm{~s}$ ). ${ }^{6}$ MAGIC's sensitivity is thus also comparable to results from other IACTs, such as HESS $(4 \sigma \sqrt{t / s})^{22}$ and VERITAS $(0.5 \sigma \sqrt{t / s}),{ }^{8}$ with the disparities attributed to different mirror size and reflectivity, resolution (strongly depending on the field of view of the central pixel), and photosensors efficiency.

\subsection{Isolated Signals: Sky Scan Test}

To test the sensitivity of the CPS to isolated optical flashes, its saturation level and different MAGIC sub-systems clock matching, dedicated observations were performed to induce fast optical flashes of known brightness into the field of view (FOV) of the CPS. This so-called sky scan test was done by slewing the MAGIC-II telescope in azimuthal direction over the star Polaris, fixing the zenith angle to that of Polaris. As the typical slewing speed of MAGIC is $\sim 4.7 \mathrm{deg} / \mathrm{s}$, stars passing through the CPS FOV $(\sim 0.1 \mathrm{deg})$ would produce optical pulses lasting $\sim 20 \mathrm{~ms}$, smeared by the optical point spread function of the telescope optics. Using this method, optical flashes of known brightness and length were recorded by the CPS, allowing us to directly verify the correlation between the voltage of a pulse and the magnitude of the stars producing such pulses. 


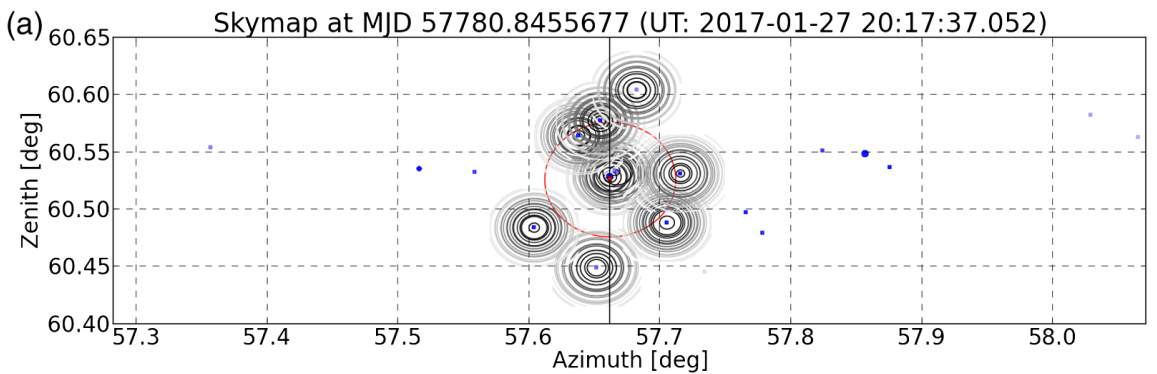

(b)

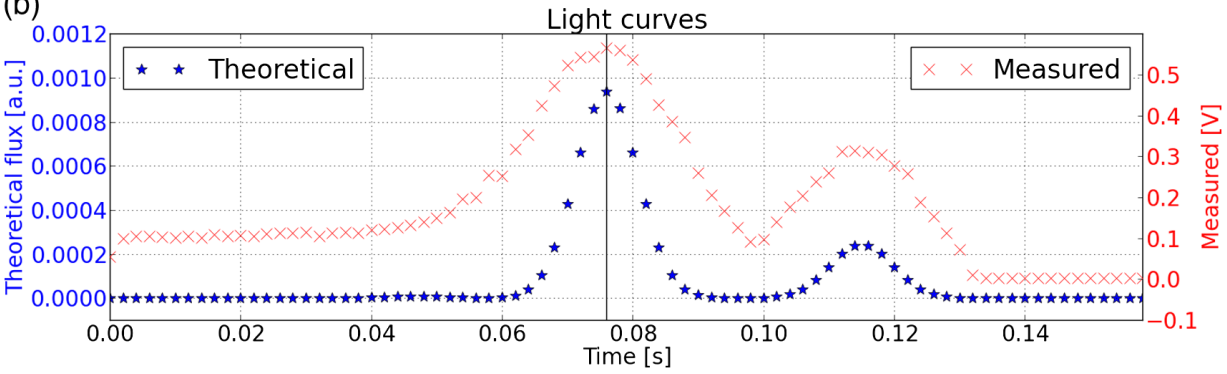

Fig. 10 Example snapshot of the sky scan test. (a) Sky view of the CPS (red circle) with stars passing through its FOV. (b) The measured voltage from the CPS (red) and the estimated photon flux (blue) showing the correlation whenever the CPS passes through bright stars.

As an example, a snapshot from this test is shown in Fig. 10. Figure 10(a) shows the sky map in horizontal coordinates, together with the position and size of the central PMT FOV (red circle) taken from the MAGIC drive system reports. ${ }^{23}$ The position of the stars (blue circles) and their magnitudes in the $\mathrm{U}$ band (proportional to the marker size) are queried from the SDSS Photometric Catalog ${ }^{24}$ within Vizier, ${ }^{25}$ using the Python package astroquery. ${ }^{26}$ A Gaussian smearing is applied to the stars near the FOV of the PMT to estimate the starlight flux collected, and the PMT voltage is averaged for 1-ms windows to reduce the impact of high-frequency noise. The correlation between the estimated and measured light curves in this test demonstrates the CPS's ability to response to the ms-duration optical signal in this test.

\subsection{Isolated Signals: Irreducible Background Evaluation}

Since IACTs do not have domes, several sources within their environment may cause optical flashes mimicking ms-duration signals in the CPS. For this reason, tens of hours of CPS data without known optical transients (corresponding to standard MAGIC dark observations) were taken to study the frequency of such (background) optical flashes. Several optical pulses with varying magnitudes and morphology were clearly detected during these observations. These optical background sources include car flashes, telescope operators activity (flashlights), satellites, and meteors (shown in Fig. 11). By storing the camera reports (PMT DC currents) with $1-\mathrm{Hz}$ frequency, MAGIC is able to efficiently identify most of these sources as background (mainly identifying moving optical transients over the camera). Meteors, on the other hand, are either too faint or travel too fast through the camera, so they are not likely to be detected by these reports. For this reason, they remain as the main background source during MAGIC operation, limiting MAGIC sensitivity to detect with high significance an optical ms transient of astrophysical origin. By studying the frequency of these meteors as a function of their magnitude, time of night, and date of observation, we are able to calculate the expected number of background events during an observation.

\section{Fast Optical Astronomy Science Cases}

The success of HiPERCAM mounted on the 10.4-m Gran Telescopio Canarias (GTC) shows the increasing interest of the scientific community to fast optical astronomy. HiPERCAM,${ }^{27}$ the most sensitive camera in the ms regime currently available, is equipped with CCD sensors capable of 

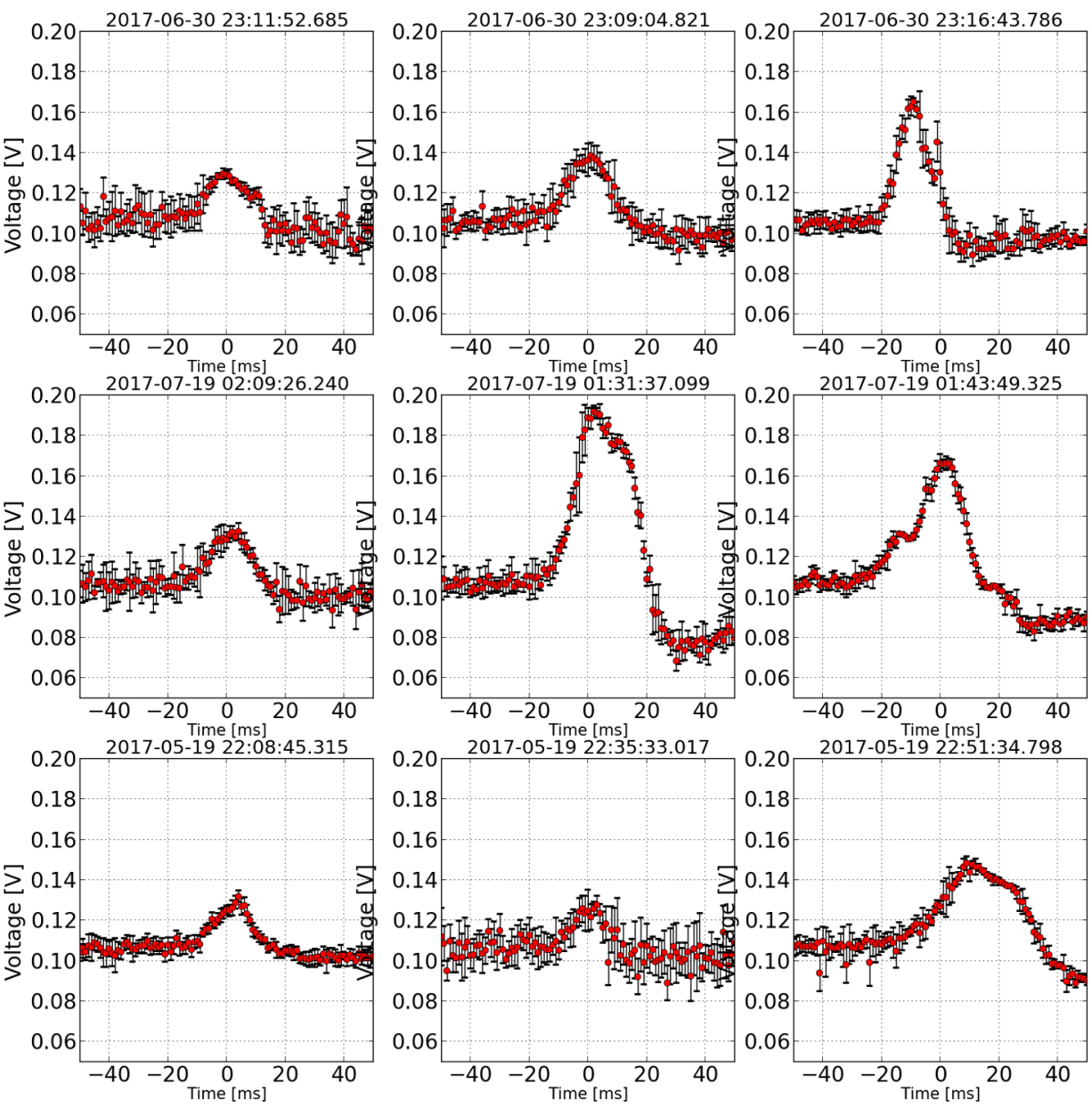

Fig. 11 Example of isolated pulses observed at dark patches of the sky, likely to be meteors (given their speed across the CPS and frequency). The time window in each light curve is $100 \mathrm{~ms}$, and the signal is binned and averaged in 1-ms time intervals to reduce the effect of high-frequency noise.

imaging at frame rates ranging from 0.001 to $1000 \mathrm{~Hz}$ over five bands in the optical simultaneously. The sensitivity of the CPS cannot compete in terms of sensitivity with HiPERCAM, given the large FOV the central pixel is integrating (strongly increasing the night-sky background rate). However, the CPS can indeed compete with HiPERCAM regarding the signal-to-noise (S/N) attained over ms timescales on bright targets (providing similar S/N as HiPERCAM). The close geographical distance between GTC and MAGIC may allow joint observations for science cases in which MAGIC is competitive. We list below some of the foreseen scientific applications of the MAGIC CPS, in order of increasing distance, from terrestrial to extragalactic sources.

- Meteor studies: The Earth continuously experiences bombardment by objects from outer space, ranging in size from large meteors capable of forming impact craters on the surface down to dust particles. Several methods have been employed to estimate the flux of these materials using space, atmospheric, and ground-based instruments. ${ }^{28}$ Observations of meteors down to magnitude 12 using the 10-m optical reflector at the Fred Lawrence Whipple Observatory have been reported. ${ }^{9}$ As mentioned in the previous section, the majority of the isolated ms-duration pulses found during the CPS observation campaigns (Fig. 11) originate from meteors traversing through MAGIC's FOV. The sensitivity to brief optical transients of the CPS and its potential wide FOV turn the MAGIC telescopes into competitive detectors for measuring the frequency of very faint meteors. MAGIC could verify the theory proposed i Ref. 29 that heating in the atmosphere during a period of elevated solar activity changes the length and maximum brightness of meteors, 
i.e., increasing solar activity leads to longer, fainter meteors and therefore lower rates by monitoring the number of isolated optical pulses observed over a particular area of the sky as a function of the observation time and the brightness of the pulse.

- Measurements of stellar angular diameters: The VERITAS telescope, another IACT array similar to MAGIC, has demonstrated that the direct measurement of stellar angular diameters with unprecedented sub-milliarcsecond resolution is possible by measuring the diffraction pattern produced by stars occulted by main belt asteroids. ${ }^{13}$ IACTs are able to provide high S/N over short observation times ( $\sim \mathrm{ms})$ for such bright targets (stars of 10 and 11th mag). By employing the CPS, MAGIC is able to directly measure stellar angular diameters of very faint stars, significantly expanding the distance at which stars may be resolved. Given the sensitivity of the MAGIC CPS (down to magnitude 13), up to one asteroid occultation per week could be predicted with enough confidence to be detected..$^{30}$ These measurements can potentially double the number of K-type stars with a stellar diameter directly measured in a few years' time. ${ }^{31}$

- Pulsar physics: Most pulsars, except for the bright Crab Pulsar, are too dim in the visible waveband to be detected by the CPS. However, optical pulsations from a transitional ms pulsar have recently been detected using instruments with comparable performance to that of MAGIC. ${ }^{32}$ This pulsar belongs to the transitional ms pulsar class which switches states between rotation-powered ms radio pulsars and accretion-powered low-mass $\mathrm{x}$-ray binaries. A new component in the $\gamma$-ray spectrum could be produced by inverse Compton scattering between the UV component of the accretion disk and the pulsar wind. This rich phenomenology motivates the use of the multiwavelength capability of MAGIC over both the optical and $\gamma$-ray regimes to search for similar sources.

- Microquasars: These systems consist of a companion donor star orbiting a compact object (which is either a neutron star or a black hole), with an accretion disk around the central compact object. ${ }^{33}$ The accretion disk may heat up due to friction between infalling particles and emit x-rays or may project narrow jets of subatomic particles at near-relativistic speed generating a strong radio emission. The disk can switch between different spectral states reflecting different modes of accretion. VHE $\gamma$-ray emission could be observed during the hard spectrum state, while during the transitional period the system exhibits rapid quasiperiodic oscillation in the optical and x-ray bands. Similar to the case of pulsars, the complex variability of the emission across the electromagnetic spectrum motivates the use of the CPS during and after the flaring state to quantify the parameters of an x-ray binary (such as the nature of the compact object or the mass of the companion). By studying correlation between optical and x-ray emission, one can shed some light on the physics behind the activity of the source in different states. ${ }^{34-36}$ The ultrafast variability that is expected from these small compact objects (unlike for example, the supermassive black hole of an active galactic nucleus) makes the timing resolution of MAGIC very valuable. Galactic microquasars, including the recently discovered MAXI J1820+070, ${ }^{37}$ are ideal targets to search for emission in various energy bands due to their relative proximity to Earth.

- Fast Radio Bursts (FRBs): Recently discovered ${ }^{38}$ FRBs are fast transient phenomena displaying short, bright pulses of radio emission. The dispersion measures of these pulses suggest their cosmological origin. To understand the emission mechanism, it would be ideal to detect these bursts at other wavelengths. Several models predict the existence of an optical burst counterpart. For example, the pulsar supergiant pulse mechanism, as seen in the Crab Pulsar, ${ }^{39}$ would have some simultaneous optical brightening associated with the radio bursts. ${ }^{40}$ Equally, there may be optical emission from magnetar giant flares. ${ }^{41}$ In fact, rapid optical flaring behavior has been seen before in a gamma-ray transient with a light curve phenomenologically similar to gamma-ray flares from magnetars. ${ }^{42}$ An optical burst detection may also provide insight into the local environment of the burst engine. ${ }^{43}$ The CPS therefore is an ideal instrument to detect these optical counterparts in case they have similar or even faster timescales. So far, MAGIC has devoted more than $25 \mathrm{~h}$ to observe the first repeating FRB121102 simultaneously in optical and VHE, including concurrent observation with other radio telescopes such as the Arecibo Observatory and the Green Bank Telescope. During this observation campaign, five radio bursts were 
detected by Arecibo. Using the CPS, MAGIC was able to set the strongest limits available on the simultaneous FRB emission both in optical and VHE. ${ }^{44}$ Recently, an FRB has been associated with a galactic magnetar, ${ }^{45}$ and the possibility of a counterpart being detected at frequencies other than radio remains open for this source. Such discovery motivates future FRB observation campaigns using the MAGIC with its CPS.

\section{Conclusion and Outlook}

As mentioned in Ref. 46 and proven by Refs. 13, 44, and 47, IACTs are competitive instruments in the field of high time resolution fast optical astronomy. Their large reflecting surfaces act as effective photon collectors while reducing the scintillation noise affecting smaller telescopes, and their ultrafast photodetectors and electronics allow faster sampling rates than CCDs. In this work, we describe the capabilities of the new CPS currently mounted on the camera of the MAGIC-II telescope. One of the goals of the MAGIC CPS is to overcome the main limitation that the previous generation CPS had: being unable to use the standard Cherenkov branch simultaneously with the optical one. After the installation of the new CPS in the MAGIC-II telescope, we were able to fulfill the following objectives.

- Standard AC branch: The CPS operates similarly to the rest of the camera during VHE $\gamma$-ray observations.

- Standard PMT anode current monitoring for the CPS: The PMT operates with its anode current within the safety limit and is protected from being damaged by bright light.

- Upgraded DC branch: The dedicated DC channel is sensitive to slow (relative to the AC branch designed to detect ns-duration flash of Cherenkov photons) light variations in a bandwidth spanning from $\mathrm{Hz}$ to $\mathrm{kHz}$, comparable to the performance of the CPS previously mounted on MAGIC-II. ${ }^{6}$

In this work, we have demonstrated the increased sensitivity of the current CPS with respect to the previous implementation. At the readout rate of $1 \mathrm{kHz}$, we were able to detect the pulsation of the Crab Pulsar within $10 \mathrm{~s}$ of observation, improving the sensitivity reported in Ref. 6. In addition, driven by the scientific interest of studying higher energy counterparts of FRBs, we report on the sensitivity of the CPS for isolated ms-scale optical flashes (see Table 2). With sensitivities in both periodic and isolated regime, MAGIC has conducted several multiwavelength studies on relevant sources with this CPS and is well positioned to play a critical role in future multiwavelength observation campaigns in the context of very-fast optical astronomy.

We have seen that the CPS captures fast optical signals from various light sources, such as meteors, car flashes, or satellites. They constitute the main background when searching for optical counterparts to astrophysical phenomena, and the current CPS is unable to distinguish between genuine fast optical events coming from astrophysical sources and these transient events. Several methods to identify these background events have been developed, mainly involving the use of the average pixel DC current reports that are stored every second during data taking. Using these methods, we are able to accurately identify car flashes, satellites, and airplanes, while some faster transients such as meteors remain unidentified.

Further improvements are possible to increase the sensitivity of the CPS. A cost-efficient solution would be to add an additional CPS to the MAGIC-I camera to allow stereoscopic optical observations. Since the two telescopes are placed $85 \mathrm{~m}$ apart, local transient signals will induce different light curves in the two CPS. Such parallax may be used to identify signals originating nearby. Unfortunately, most meteors (the main source of irreducible background) will travel too high in the atmosphere to be effectively resolved using parallax. Additionally, as the effective observation time is doubled, the second CPS will increase the sensitivity by a factor of $\sqrt{2}$, assuming that the main source of noise is the night-sky background. A more elaborate improvement can be achieved by turning the entire central cluster into one 7-pixel composite CPS, incorporating a veto system with the surrounding six pixels, similar to the method reported in Ref. 46. In this way, any meteor signal appearing in the central pixel would also leave an imprint in a minimum of 2 adjacent pixels and can be readily identified by measuring the timing offset across different pixels between these signals. 


\section{Acknowledgments}

We acknowledge the financial support of the German Federal Ministry of Education and Research (BMBF), the German Research Foundation (DFG) and The Max Planck Society for the Advancement of Science (MPG), and the European Regional Development Fund (ERDF) under the Spanish Ministry of Economy and Competitiveness (MINECO) Grant FPA201785668. For this paper, the authors hereby declare no relevant financial interests and have no other potential conflicts of interest to disclose.

\section{References}

1. J. Aleksic et al., "The major upgrade of the MAGIC telescopes, Part I," Astropart. Phys. 72, 61-75 (2016).

2. J. Osborn et al., "Atmospheric scintillation in astronomical photometry," Mon. Not. R. Astron. Soc. 452, 1707-1716 (2015).

3. M. Daniel, "Non-gamma-ray applications of TeV telescopes," in Proc. 36th Int. Cosmic Ray Conf., Wisconsin, Madison, p. 358 (2019).

4. R. W. Lessard et al., "Search for pulsed TeV gamma-ray emission from the Crab Pulsar?" Astrophys. J. 531, 942-948 (2000).

5. E. O. Wilhelmi et al., "Determination of the night sky background around the crab pulsar using its optical pulsation," Astropart. Phys. 22, 95-102 (2004).

6. F. Lucarelli et al., "The central pixel of the MAGIC telescope for optical observations," Nucl. Instrum. Methods Phys. Sec. A 589(3), 415-424 (2008).

7. J. Hinton et al., "Precision measurement of optical pulsation using a Cherenkov telescope," Astropart. Phys. 26, 22-27 (2006).

8. S. Griffin, D. Hanna, and A. Gilbert, "Searching for fast optical transients using VERITAS Cherenkov telescopes," in Proc. 32th Int. Cosmic Ray Conf., Beijing, China, Vol. 9, pp. 321323 (2012).

9. A. F. Cook et al., "Flux of optical meteors down to $M_{\mathrm{pg}}=+12$," Mon. Not. R. Astron. Soc. 193, 645-666 (1980).

10. D. Eichler and G. Beskin, "Optical search for extraterrestrial intelligence with Air Cerenkov telescopes," Astrobiology 1, 489-493 (2001).

11. A. U. Abeysekara et al., "A search for brief optical flashes associated with the SETI target KIC 8462852," Astrophys. J. Lett. 818(2), L33 (2016).

12. V. Acciari et al., "Optical intensity interferometry observations using the MAGIC imaging atmospheric Cherenkov telescopes," Mon. Not. R. Astron. Soc. 491(2), 1540-1547 (2020).

13. W. Benbow et al., "Direct measurement of stellar angular diameters by the VERITAS Cherenkov telescopes," Nat. Astron. 3, 511-516 (2019).

14. D. B. Tridon et al., "Performance of the camera of the MAGIC II telescope," in Proc. 31st ICRC, Lodz, Poland, arXiv:0906.5448 (2009).

15. D. H. Staelin, C. Edward, and C. Reifenstein, "Pulsating radio sources near the Crab Nebula," Science 162, 1481-1483 (1968).

16. J. M. Comella et al., "Crab Nebula Pulsar NP 0532," Nat. Lett. 221, 453-454 (1969).

17. N. Lewandowska, "The pulsar in the Crab Nebula," in Proc. Front. Res. Astrophys., Mondello, Palermo, Italy, arXiv:1511.05211v1 (2014).

18. A. G. Lyne, R. S. Pritchard, and F. Graham-Smith, "Data were collected from an online database, and originated from Lyne," Mon. Not. R. Astron. Soc. (MNRAS), A. G. Lyne, R. S. Pritchard, and F. Graham-Smith, Eds., 265, 1003 (1993).

19. A. Lyne, R. Pritchard, and F. Smith, "23 years of Crab Pulsar rotational history," Mon. Not. R. Astron. Soc. 265, 1003-1012 (1993).

20. C. Germanà et al., "Aqueye optical observations of the Crab Nebula Pulsar," Astron. Astrophys. 548, A47 (2012).

21. M. López, "Astronomía Gamma con el Telescopio MAGIC: Observaciones de la Nebulosa y Pulsar del Cangrejo," PhD Thesis, Universidad Complutense de Madrid (2007).

22. A. Franzen et al., "Optical observations of the Crab Pulsar using the first H.E.S.S. Cherenkov telescope," in Proc. 28th ICRC, Trukuba, Japan, Vol. 5, pp. 2987-2990 (2003). 
23. T. Bretz et al., "The drive system of the major atmospheric gamma-ray imaging Cherenkov telescope," Astropart. Phys. 31(2), 92-101 (2009).

24. S. Alam et al., "The eleventh and twelfth data releases of the Sloan Digital Sky survey: final data from SDSS-III," Astrophys. J. Suppl. 219(1), 12 (2015).

25. F. Ochsenbein et al., "The VizieR database of astronomical catalogues," Astron. Astrophys. Suppl. Ser. 143(1), 23-32 (2000).

26. A. Ginsburg et al., "Astroquery: an astronomical web-querying package in python," Astron. J. 157(3), 98 (2019).

27. V. Dhillon et al., "First light with HiPERCAM on the GTC," Proc. SPIE 10702, 107020L (2018).

28. M. Zolensky et al., "Flux of extraterrestrial materials," in Meteorites and the Early Solar System II, D. S. Lauretta and H. Y. McSween, Eds., Vol. 943, pp. 869-888, University of Arizona Press, Tucson, Arizona (2006).

29. M. Campbell-Brown, "Solar cycle variation in radar meteor rates," Mon. Not. R. Astron. Soc. 485, 4446-4453 (2019).

30. T. Hassan and M. Daniel, "Proving the outstanding capabilities of IACTs in high time resolution optical astronomy," in Proc. 36th ICRC, Madison, arXiv:1908.033938 (2019).

31. F. Spada et al., "The radius discrepancy in low-mass stars: single versus binaries," Astrophys. J. 776(2), 87 (2013).

32. F. Ambrosino et al., "Optical pulsations from a transitional millisecond pulsar," Nat. Astron. 1(12), 854-858 (2017).

33. I. F. Mirabel and L. Rodríguez, "Microquasars in our galaxy," Nature 392, 673-676 (1998).

34. D. Psaltis, T. Belloni, and M. Klis, "Correlations in quasi-periodic oscillation and noise frequencies among neutron star and black hole x-ray binaries," Astrophys. J. 520(1), 262-270 (1999).

35. P. Uttley, "The relationship between optical and x-ray variability in Seyfert Galaxies," ASP Conf. Ser. 360, 101 (2006).

36. M. Shidatsu et al., "X-ray and optical monitoring of state transitions in MAXI J1820+070," Astrophys. J. 874, 183 (2019).

37. L. Zampieri et al., "Low-frequency optical QPO in MAXI J1820+070 detected with IFI +IQUEYE@Galileo,” Astronomer's Telegram (11824) (2018).

38. D. R. Lorimer et al., "A bright millisecond radio burst of extragalactic origin," Science 318(5851), 777-780 (2007).

39. M. Lyutikov, L. Burzawa, and S. Popov, "Fast radio bursts as giant pulses from young rapidly rotating pulsars," Mon. Not. R. Astron. Soc. 462, 941-950 (2016).

40. A. Shearer et al., "Enhanced optical emission during crab giant radio pulses," Science 301, 493-495 (2003).

41. M. Lyutikov and D. Lorimer, "How else can we detect fast radio bursts?" Astrophys. J. Lett. 824(2), L18 (2016).

42. A. Stefanescu et al., "Very fast optical flaring from a possible new Galactic magnetar," Nature 455, 503-505 (2008).

43. B. Metzger, E. Berger, and B. Margalit, "Millisecond Magnetar birth connects FRB 121102 to superluminous supernovae and long-duration gamma-ray bursts," Astrophys. J. 841(1), 14 (2017).

44. V. Acciari et al., "Constraining very-high-energy and optical emission from FRB 121102 with the MAGIC telescopes," Mon. Not. R. Astron. Soc. 481, 2479-2486 (2018).

45. C. D. Bochenek et al., "A fast radio burst associated with a Galactic magnetar," https://arxiv .org/abs/2005.10828 (2020).

46. C. Deil et al., "Capability of Cherenkov telescopes to observe ultra-fast optical flares," Astropart. Phys. 31, 156-162 (2009).

47. J. Holder and R. S. Lynch, "VERITAS observations of fast radio bursts," in Proc. 36th Int. Cosmic Ray Conf., p. 698 (2020).

Biographies of the authors are not available. 